\title{
RELAÇÃo do PERÍOdo DE PERMANÊNCIA EM VENTILAÇÃo MECÂNICA INVASIVA COM O GÊNERO E A IDADE DOS PACIENTES DA UTI DO HOSPITAL MUNICIPAL DE RIO VERDE
}

\author{
Leonardo Squinello Nogueira Veneziano ${ }^{1}$ \\ Millene Leite Oliveira ${ }^{2}$ \\ Nulciene Firmino de Freitas ${ }^{3}$ \\ Getulio Antonio de Freitas Filho ${ }^{4}$ \\ Rejane Maria Cruvinel Cabral ${ }^{5}$ \\ Roberto Dias ${ }^{6}$ \\ Lya Carla Manso Miranda ${ }^{7}$ \\ Katia Silveira Ferreira ${ }^{8}$ \\ Renata Nascimento Silva ${ }^{9}$ \\ Fernando Duarte Cabra ${ }^{10}$
}

Resumo - A Insuficiência Respiratória é uma patologia comum dentro de uma Unidade de Terapia Intensiva (UTI), levando os pacientes a necessitarem de Ventilação Pulmonar Mecânica.Essa pesquisa objetiva-se a avaliar o período de permanência em ventilação mecânica invasiva em relação ao gênero e a idade dos pacientes. Trata-se de um estudo cuja amostra foi composta por 50 prontuários, no período de março a maio de 2015 na UTI do Hospital Municipal de Rio Verde. O presente estudo apresentou os seguintes resultados em relação ao gênero dos 50 participantes da pesquisa,não foram encontradas diferenças estatisticamente significativas $(P=0,9184)$ entre os dois gêneros. no entanto em relação à faixa etária, 0 presente estudo não obteve alteração estatisticamente significativa entre os grupos,contudo em relação a permanência em VMI demonstrou diferenças estatisticamente significativas $(P=0,0413$ ) entre os grupos 40 a 59 anos e 60 a 80 anos. Conclui-se portanto que não houve diferença no período de ventilação mecânica relacionada ao gênero.

Palavras-chave: Insuficiência respiratória; Ventilação mecânica; Permanência; Sexo; Idade.

\footnotetext{
${ }^{1}$ Faculdade Objetivo Rio Verde, Brasil. E-mail: leosnv@yahoo.com.br.

2 Faculdade Objetivo Rio Verde, Brasil. E-mail: kakaua_@hotmail.com.

${ }^{3}$ Faculdade Objetivo Rio Verde, Brasil. E-mail: nulciene@faculdadeobjetivo.com.br.

${ }^{4}$ Faculdade Objetivo Rio Verde, Brasil. E-mail: getulio@faculdadeobjetivo.com.br.

${ }^{5}$ Faculdade Objetivo Rio Verde, Brasil. E-mail: rejane@faculdadeobjetivo.com.br.

${ }^{6}$ Faculdade Objetivo Rio Verde, Brasil. E-mail: fisiodias@uol.com.br.

${ }^{7}$ Faculdade Objetivo Rio Verde, Brasil. E-mail: lya@faculdadeobjetivo.com.br.

${ }^{8}$ Faculdade Objetivo Rio Verde, Brasil. E-mail: katiafisio1@bol.com.br.

${ }^{9}$ Faculdade Objetivo Rio Verde, Brasil. E-mail: renata@ faculdadeobjetivo.com.br.

${ }^{10}$ Faculdade Objetivo Rio Verde, Brasil. E-mail: fernandofisio2@hotmail.com.
} 